\begin{tabular}{|r|c|c|c|c|c|}
\hline Revista Clío América & ISSN: 1909-941X & Vol. 12 & No. 23 & enero - junio de 2018 & 52 - 61 \\
\hline
\end{tabular}

\title{
Relación entre liderazgo e innovación en las pymes de la comuna once de Medellín
}

\author{
Relationship between leadership and innovation \\ in the smes of la comuna eleven de Medellin
}

\section{c) () (-) \\ BY NE SA}

RESUMEN: El liderazgo y la innovación son elementos determinantes en el éxito empresarial; ellos hacen parte importante y significativa del proceso administrativo, y son condicionantes para llevar a las empresas a la consecución de sus objetivos. Este artículo busca determinar cómo el estilo de liderazgo incide en la adopción de procesos de innovación al interior de las PYMES de la comuna 11 de la ciudad de Medellín, a partir de la identificación de los estilos de liderazgo, y su relación con los procesos de innovación desarrollados dentro de las organizaciones; se aplicó el enfoque cuantitativo y se consideraron como técnicas de recolección de datos la encuesta y la observación directa. Para la instrumentalización de los datos se utilizó una secuencia de análisis deductivo, secuencial, probatorio que analiza la realidad objetiva. Además, la codificación de la información se realizó con base en las categorías predefinidas, a partir de la pregunta de investigación. Entre las principales conclusiones se identificó que el estilo participativo es el que potencia el desarrollo de las personas; lo que propicia la innovación, la eficiencia y productividad organizacional.

Palabras clave: innovación - liderazgo - Pyme - estilos de liderazgo - organizaciones.

JEL: M100

ABSTRACT: Leadership and innovation are decisive elements in business success; they are an important and significant part of the administrative process, and they are determining factors in leading companies to achieve their objectives. This article seeks to determine how the style of leadership affects the adoption of innovation processes within the SMEs of the 11th district of the city of Medellin, based on the identification of leadership styles, and their relationship with the processes of innovation developed within organizations; the quantitative approach was applied and survey and direct observation were considered as data collection techniques. For the instrumentalization of the data, a sequence of deductive, sequential, probatory analysis that analyzes the objective reality was used. In addition, the coding of the information was made based on the predefined categories, based on the research question. Among the main conclusions, it was identified that the participatory style is what promotes the development of people; what promotes innovation, efficiency and organizational productivity.

Keywords: Innovation - Leadership - SMEs - Leadership Styles - Organizations.

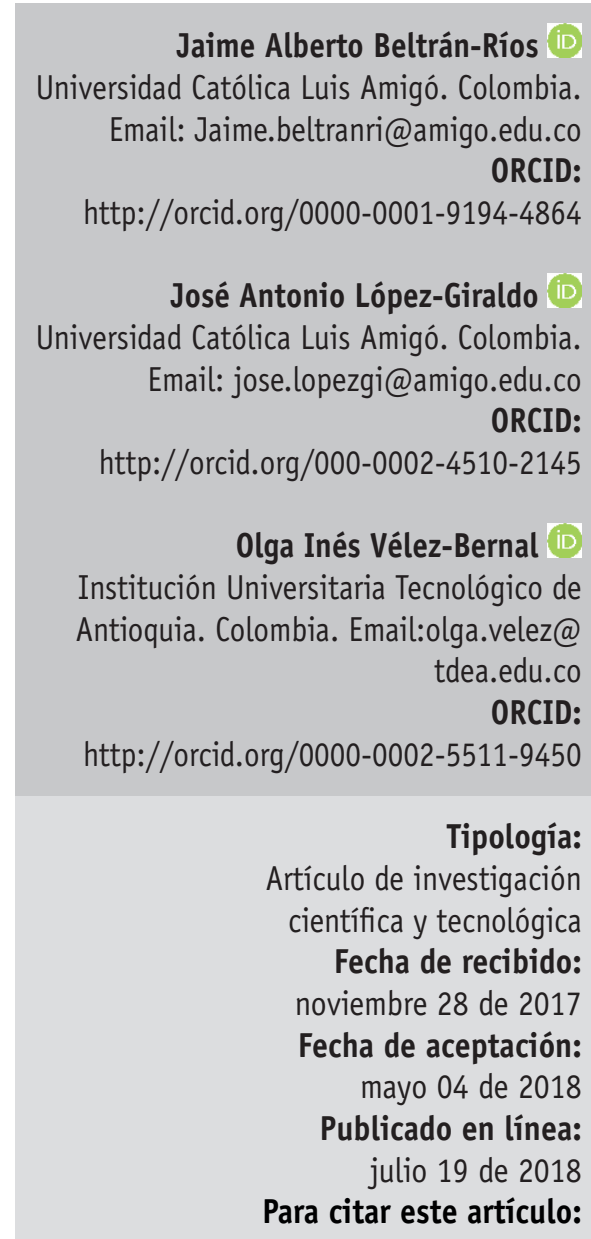

Beltrán, R. J., López, G. J. y Vélez B. 0. (2018). Relación entre liderazgo e innovación en las pymes de la comuna once de Medellín. Clío América, 12(23),

p. 52-61.

Doi: $10.21676 / 23897848.2616$ 


\section{INTRODUCCIÓN}

Las dinámicas sociales y económicas en los países están ocasionando modificaciones estratégicas para incentivar ideas de innovación, que posibiliten una idoneidad dentro de las competencias de mercado con el resto del mundo. La innovación se ha convertido en uno de los instrumentos fundamentales dentro de las empresas que quieren seguir siendo competitivas, y además socialmente responsables, en un entorno cada vez más complejo y cambiante. Con todo, la innovación no es un concepto fácil de entender; muchas empresas, e incluso académicos, todavía desconocen su significado y la manera en que puede afectar su evolución (González y Martínez, 2014). Los teóricos españoles Pons y Ramos (2012) aseguran que, para una complementación total de la innovación, son indispensables elementos de creatividad que soporten el proceso constitutivo de ideas reformadoras; por lo cual, el clima organizacional, como factor holístico, da cabida para una contribución de la creatividad en los empleados y así obtener un "clima de innovación estimulante" (p. 82). La idea de innovación, dentro de las dinámicas empresariales, favorece al manejo adecuado de las bases económicas con el propósito de no afrontar una crisis extensa. Ante esto, se esclarece que la innovación dentro de las organizaciones modernas "se caracterizan por estar orientadas hacia la creatividad y el cambio, por apoyar la independencia de sus empleados en la búsqueda de nuevas ideas" (pp. 82-83). La anterior cita aborda la necesidad de las ideas creativas por el constante desafío de las organizaciones ante la alta competitividad de mercados; es decir, aquellas estructuras empresariales deben adoptar más desarrollos de mayor flexibilidad, creatividad e innovación.

Es indudable la importancia que tiene el liderazgo en la implementación de procesos innovadores al interior de las empresas, ya que estos propician el crecimiento individual y colectivo de los equipos de trabajo a partir del empoderamiento y procesos de formación. García (2015) a partir de una revisión de 35 artículos y 20 libros, sobre las teorías organizacionales, propone un modelo de liderazgo determinado por la relación existente del liderazgo con cada una de las dimensiones del proceso: el ambiente, asociado a la situación específica y las estrategias; la situación específica, asociada a las estrategias, al líder y al ambiente; las estrategias asociadas con la organización, el líder, los resultados, la situación específica y el cliente y la comunidad (p. 70).

Otra de las características estudiadas ha sido el análisis de la influencia que ejercen los líderes sobre el entorno y sus procesos; para ilustrarlo, Escandon y Hurtado (2016) propusieron una investigación sobre la influencia del liderazgo en el desempeño de las empresas exportadoras colombianas; se basaron en determinar qué tipo de liderazgo incide en su alta productividad, partiendo de que según Warrick (como se citó en Escandon y Hurtado, 2016), los estilos de liderazgo se identifican por sus características, su filosofía y el conjunto de habilidades de gestión propias. Las características del estilo de liderazgo describen la importancia de un líder para obtener rendimiento. La filosofía describe los supuestos del líder sobre las personas y el papel del líder. Las habilidades incluyen las capacidades de gestión propias de un estilo particular. (p. 139), a partir de tres estilos de liderazgo para la realización del estudio, autocrático, democrático y liberal; en el primero el líder toma sus propias decisiones sin incluir a los demás; el segundo toma en cuenta la opinión de sus colaboradores y los incluye en las decisiones, y el tercero le da libertad y autonomía a sus subordinados e interviene solo en situaciones de necesidad (Escandon y Hurtado, 2016).

Dadas las dificultades que se observan en los procesos de innovación, realizados por parte de las empresas, como respuesta a los desafíos que les plantean los cambios del entorno, se propone como pregunta central de este trabajo ¿Cómo incide $\mathrm{El}$ estilo de liderazgo en la adopción de procesos de innovación en las PYMES de la comuna 11 de la ciudad de Medellín? Dentro de los estilos de liderazgo que facilitan los procesos de innovación se encuentra el liderazgo transaccional, desarrollado por Bernard Bass a finales de los años ochenta del siglo XX; este modo tomó fundamentos vitales de estilos como el carismático y transformacional, desarrollados por House y Burns (como se citó en González y Carrasquillo, 2013) se visualiza como el intercambio entre el líder y su grupo de trabajo seguidores - donde estos reciben un valor a cambio 
de su trabajo (costo - beneficio). El líder transaccional identifica las necesidades y anhelos de sus seguidores, por lo que aquel dialoga con su grupo expresando claramente la manera como se pueden suplir las necesidades a cambio de que cumplan con los objetivos específicos. Ante esto, los seguidores reciben bonificaciones por su desempeño dentro de las dinámicas laborales. El carisma 0 influencia idealizada es el factor por antonomasia del liderazgo transaccional, puesto que este hace referencia a la capacidad del líder para anunciar una misión y ganar la confianza de su grupo. Por su parte, la inspiración se define como la facultad del líder para comunicar la visión de la organización; provoca a los seguidores confianza y entusiasmo para la realización de los objetivos. Y por último, la estimulación intelectual es la capacidad del líder para que sus seguidores piensen de manera innovadora y creativa (Pons y Ramos, 2012). Por lo tanto, "algunas de las ventajas de este estilo de liderazgo es que se concentran en el presente y son muy buenos para lograr que la organización funcione eficientemente" (Berdecía, González y Carrasquillo, 2013, p. 23). El liderazgo transaccional junto a sus componentes de eficacia brindan para la era del conocimiento y la información una manera novedosa de concebir las dinámicas laborales que se expresan dentro de las organizaciones, dado que el entorno cambiante en el que está inserto las empresas obliga a los líderes a ofrecer respuestas inmediatas y adecuadas con el fin de sobrevivir al vertiginoso mundo del mercado. Ante esto, las organizaciones empresariales, según Serrano y Portalanza (2014), deben reinventar y reconstituir la noción de liderazgo; esto quiere decir, la necesidad de adherir nuevas características en los líderes para que dichas organizaciones se conviertan en inteligentes $y$, además, que el clima organizacional sea beneficioso tanto para el líder como para los seguidores con el objetivo de que estos adquieran una potenciación en los componentes del liderazgo transaccional $y$, en consecuencia, se ofrezca la apertura de ideas innovadoras.

Según Yeung, Nason y Von (2000) los factores que posibilitan y vigorizan la capacitación de los miembros de las empresas son en total doce. No obstante, la característica esencial de estos componentes es que se dirigen, esencialmente, al líder como símbolo de innovación y potenciación de las dinámicas empresariales:

1. El líder entrena y forma a los miembros.

2. El diálogo como elemento funcional.

3. Capacitación a los miembros.

4. Búsqueda de nuevas soluciones.

5. Experimentación de nuevas ideas.

6. Sobrepasa el plano personal para hallar propuestas equitativas.

7. Difunde información.

8. Aprendizaje de los errores.

9. Desarrollo de la visión empresarial.

10. Ofrece tiempo para el aprendizaje.

11. Brinda medios para el triunfo de otros.

12. Comparte premios y logros (p. 193).

Estas doce cualidades expuestas se congregan y concentran en tres categorías esenciales y vitales para la consolidación del conocimiento en la organización $y$, por consiguiente, la aprehensión de la innovación en la misma:

1. Entrenador: se describe como el líder que desea capacitar a su grupo de trabajo, con el fin de que este elabore adecuadamente su labor.

2. Experimentador: se describe como el líder que desarrolla y busca nuevas soluciones para mejorar el funcionamiento de la organización.

3. Visionario: es la capacidad del líder para plantear retos y objetivos.

Tanto las doce características del líder como estas tres categorías fueron esenciales para el análisis del liderazgo y la innovación dentro de los PYMES de la comuna 11 de la ciudad de Medellín.

La noción de innovación es de carácter fundamental para la organización, puesto que aporta unos beneficios para la competitividad empresarial y, también, favorece a la subsistencia de la empresa dentro del entorno cambiante del mercado. La organización empresarial que incluye la innovación le permite un desarrollo potencial dentro del mercado, a través de la realización de un proceso novedoso; esto refiere a que la innovación, como elemento fundamental del liderazgo, proporciona una mejor posición de la 
empresa en las dinámicas de mercado y un mejor comportamiento en este mismo. La innovación empresarial es, entonces, la voluntad de experimentar, conocer y correr riesgos para obtener un manejo adecuado entre los miembros de la empresa y, así, obtener el resultado de los objetivos propuestos por el líder.

Con los resultados de este estudio se busca determinar la incidencia del estilo de liderazgo en la adopción de procesos de innovación en las PYMES de la comuna 11 de la ciudad de Medellín, a partir del muestreo realizado a 386 colaboradores donde se identificó que el estilo de liderazgo participativo es el que potencia el desarrollo de la innovación en la organización.

\section{METODOLOGÍA}

El trabajo se enmarca en la corriente de la investigación cuantitativa; este tipo de investigación es deductiva; "utiliza la recolección de datos para probar hipótesis con base en la medición numérica y el análisis estadístico, con el fin de establecer pautas de comportamiento y probar teorías", (Hernández, Fernández y Baptista, 2014).

El enfoque cuantitativo es secuencial y probatorio. Cada etapa precede a la siguiente y no podemos "brincar" o eludir pasos. El orden es riguroso, aunque desde luego, podemos redefinir alguna fase. Parte de una idea que va acotándose $y$, una vez delimitada, se derivan objetivos y preguntas de investigación, se revisa la literatura y se construye un marco o una perspectiva teórica. De las preguntas se establecen hipótesis y determinan variables; se traza un plan para probarlas (diseño); se miden las variables en un determinado contexto; se analizan las mediciones obtenidas utilizando métodos estadísticos, y se extrae una serie de conclusiones, (Hernández, Fernández y Baptista, 2014).

La construcción del protocolo para el desarrollo del estudio se enfoca, esencialmente, en dos factores: la significación de las fuentes y procedimientos para la toma de datos y los distintos métodos para dicha recolección y estudio de datos (Pons y Ramos,
2012). El compendio de datos tomados se adquieren a través de las distintas fuentes que requiere el estudio: encuestas, observaciones y estudio de documentos enfocados en el objeto de estudio de esta investigación.

Para este trabajo de investigación, la unidad de análisis fue definida en una muestra representativa de las pymes de la comuna 11 de la ciudad de Medellín.

La muestra de este proyecto se ejecutó con 386 colaboradores de las PYMES de la comuna 11 (Laureles) de la ciudad de Medellín pertenecientes a los siguientes sectores: transporte, tecnología, educación, alimentos, hoteles. La muestra está compuesta por 174 hombres equivalente al $45 \%$ de la población y 212 mujeres equivalente al $55 \%$.

La edad promedio de los encuestados es de 38 años. El promedio de antigüedad en la empresa es de 6 años.

Los cargos ocupados por los encuestados se agrupan así: $58 \%$ personal de base, $31 \%$ Personal administrativos, $6 \%$ cargos de jefatura, y el $5 \%$ cargos de supervisión.

El $39 \%$ tienen contrato laboral a término fijo, el 52 $\%$ contrato temporal y el $9 \%$ contrato a término indefinido.

El grado de escolaridad se define así: $17 \%$ universitario, $32 \%$, técnico y tecnológico, $46 \%$ bachilleres y el $5 \%$ educación básica.

La construcción del cuestionario con el propósito de extraer los datos para la elaboración del estudio (tablas y gráficos) toma un número de preguntas que hacen referencia al modelo organizacional de la empresa. El cuestionario propone cuatro respuestas, siendo 1; Totalmente en desacuerdo, 2; En desacuerdo, 3; de acuerdo, 4; Muy de acuerdo.

Las variables empleadas para identificar el estilo de liderazgo fueron:

- Influencia del líder sobre la conducta del trabajador 
- Capacidad de motivación del líder

- Promueve el desarrollo intelectual del trabajador

- Reconocimiento Individual del trabajador

- Valoración de la productividad del trabajador

- Pasividad del líder en la toma de decisiones

- Influye muy poco en los logros y objetivos de la empresa

- Información compartida

- Participación del empleado

- Proporciona compensación justa y equitativa

El cuestionario plantea cuatro alternativas de respuesta: 1) Nunca, 2.) Rara Vez, 3.) A Veces, 4.) Siempre.

\section{RESULTADOS}

En la Tabla 1 puede identificarse que la adopción de procesos para incluir ideas innovadoras dentro de las PYMES de la comuna 11 de Medellín puede tener un mejor fortalecimiento, puesto que sólo tres variables (generación de ideas, estimulación de la creatividad y uso de herramientas de generación de ideas) superan el $50 \%$ en estar "de acuerdo" con la manera como el líder de la organización empresarial proporciona actitud y carácter con el propósito de influir en sus seguidores y, así, poder afianzar ideas de innovación dentro de su empresa. Por otra parte, hubo dos variables (sistematización de vigilancia y filtración de buenas ideas) que superan el $50 \%$ en la respuesta "En desacuerdo"; esto da pie para crear una inestabilidad entre líder y seguidores con el objetivo de alcanzar las metas y proyectos que, posteriormente, se constituyan ejercicios de innovación.

En la Tabla 2 se puede dar por sentado que el estilo más valorado en la encuesta es el estilo participativo; esto quiere decir que el jefe, o líder, tiene una influencia y comunicación oportuna y adecuada con su grupo de trabajo. Esto favorece, entonces, al comportamiento de sus seguidores para un manejo laboral apropiado para el alcance de metas. Además, se denota que hay un componente del estilo liberal de liderazgo que tiene un vínculo fuerte con el participativo, y es el completo predominio del líder con la realización de objetivos y con el cumplimiento de logros de la empresa.

Los estilos de liderazgo están insertos en la Tabla 2. Sin embargo, es indispensable anexar las preguntas desarrolladas en la encuesta a los PYMES para una mejor claridad de resultados: 1.) ¿El estilo de liderazgo de su jefe influye positivamente en su comportamiento laboral? 2.) ¿Percibe en su jefe una persona motivadora? 3.) ¿Su jefe permite que se desarrollen procesos de aprendizaje en el área? 4.) ¿Su jefe realiza reconocimientos individuales de desempeño? 5.) ¿Su jefe maneja indicadores de productividad? 6.) ¿Su jefe es pasivo al momento de tomar decisiones? 7.) ¿Su jefe influye muy poco en los logros y objetivos de la empresa? 8.) ¿Su jefe comparte la información de la empresa con los trabajadores? 9.) ¿Su jefe permite la participación de los trabajadores en la toma de decisiones? 10.) ¿Su jefe genera una compensación justa y equitativa por las labores realizadas?

En la Tabla 3 los resultados obtenidos indican que el liderazgo participativo y el autocrático fueron los predominantes en el estudio realizado; en los gráficos de correlaciones se muestra que hay una relación proporcional entre el grado de innovación de las empresas y el liderazgo participativo, pero también existe una relación entre el grado de innovación de las empresas y el liderazgo autocrático. Es decir que en las empresas que son innovadoras predomina el liderazgo participativo, pero también hay presencia del liderazgo autocrático. (Figura 1, Figura 2, Figura 3) 
Tabla 1.

Adopción de procesos de innovación en las PYMES de la comuna 11 de Medellín

\begin{tabular}{|l|c|c|c|c|}
\hline \multicolumn{1}{|c|}{ CLIMA INNOVACIÓN } & $\begin{array}{c}\text { Totalmente en } \\
\text { desacuerdo }\end{array}$ & $\begin{array}{c}\text { En desa- } \\
\text { cuerdo }\end{array}$ & De acuerdo & $\begin{array}{c}\text { Totalmente } \\
\text { de acuerdo }\end{array}$ \\
\hline Generación de ideas. & $0 \%$ & $2,59 \%$ & $85,50 \%$ & $11,91 \%$ \\
\hline $\begin{array}{l}\text { Estimulación de creatividad del grupo } \\
\text { de trabajo. }\end{array}$ & $3,62 \%$ & $17,61 \%$ & $77,72 \%$ & $1,05 \%$ \\
\hline $\begin{array}{l}\text { Sistematización de vigilancia tecno- } \\
\text { lógica. }\end{array}$ & $24,35 \%$ & $59,07 \%$ & $16,58 \%$ & $0 \%$ \\
\hline $\begin{array}{l}\text { Uso de herramientas de generación de } \\
\text { ideas. }\end{array}$ & $12,43 \%$ & $24,87 \%$ & $56,47 \%$ & $6,23 \%$ \\
\hline Filtrar buenas ideas. & $3,10 \%$ & $59,06 \%$ & $16,58 \%$ & $21,26 \%$ \\
\hline
\end{tabular}

Fuente: elaboración propia.

Tabla 2.

Estilos de liderazgo dentro de los PYMES de la comuna 11 de Medellín.

\begin{tabular}{|l|r|r|r|r|}
\hline \multicolumn{1}{|c|}{ ESTILOS DE LIDERAZGO } & Nunca & Rara vez & A veces & Siempre \\
\hline Autocrático & $10,36 \%$ & $0 \%$ & $16,58 \%$ & $73,06 \%$ \\
\hline Participativo & $0 \%$ & $12,95 \%$ & $21,25 \%$ & $65,80 \%$ \\
\hline Participativo & $1,56 \%$ & $10,88 \%$ & $76,68 \%$ & $10,88 \%$ \\
\hline Participativo & $0 \%$ & $10,62 \%$ & $12,18 \%$ & $77,20 \%$ \\
\hline Autocrático & $0,53 \%$ & $18,39 \%$ & $71,24 \%$ & $9,84 \%$ \\
\hline Liberal & $15,03 \%$ & $66,32 \%$ & $18,65 \%$ & $0 \%$ \\
\hline Liberal & $25,90 \%$ & $60,90 \%$ & $12,69 \%$ & $0,51 \%$ \\
\hline Participativo & $0 \%$ & $7,77 \%$ & $13,98 \%$ & $78,25 \%$ \\
\hline Participativo & $1,29 \%$ & $2,35 \%$ & $14,24 \%$ & $82,12 \%$ \\
\hline Participativo & $4,92 \%$ & $26,68 \%$ & $47,15 \%$ & $21,29 \%$ \\
\hline
\end{tabular}

Fuente: elaboración propia. 


\section{Tabla 3.}

Estadísticos descriptivos y coeficientes de correlación entre la innovación y los estilos de liderazgo en las PYMES de la comuna 11 de Medellín

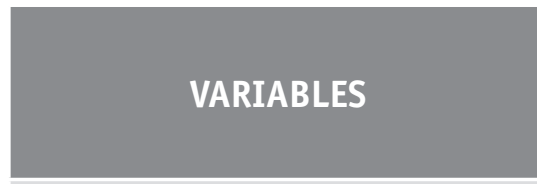

1. Clima de innovación

2. Liderazgo participativo

3. Liderazgo autocrático

4. Liderazgo liberal
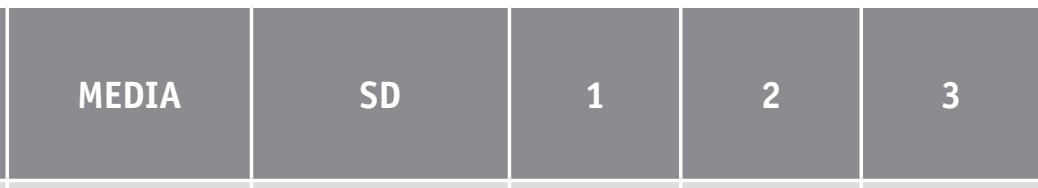

\section{2,66}

0,726

3,27

1,96

3,41

\section{0,726}

3,41

0,748

0,705

0,610

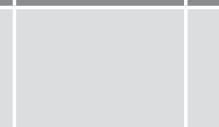

0,832

0,768

0,863

$-0,249 \quad-0,330$
$-0,292$

\section{Figura 1.}

De correlaciones entre las variables estilos de liderazgo e innovación

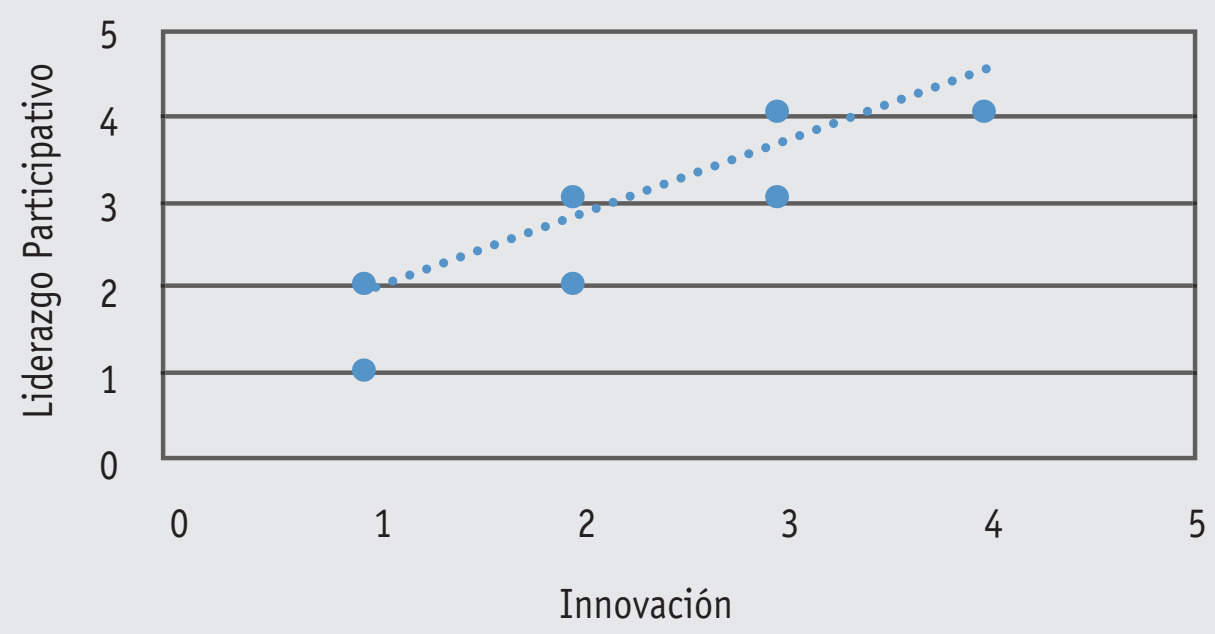

Fuente: elaboración propia. 
Figura 2.

Liderazgo autocrático

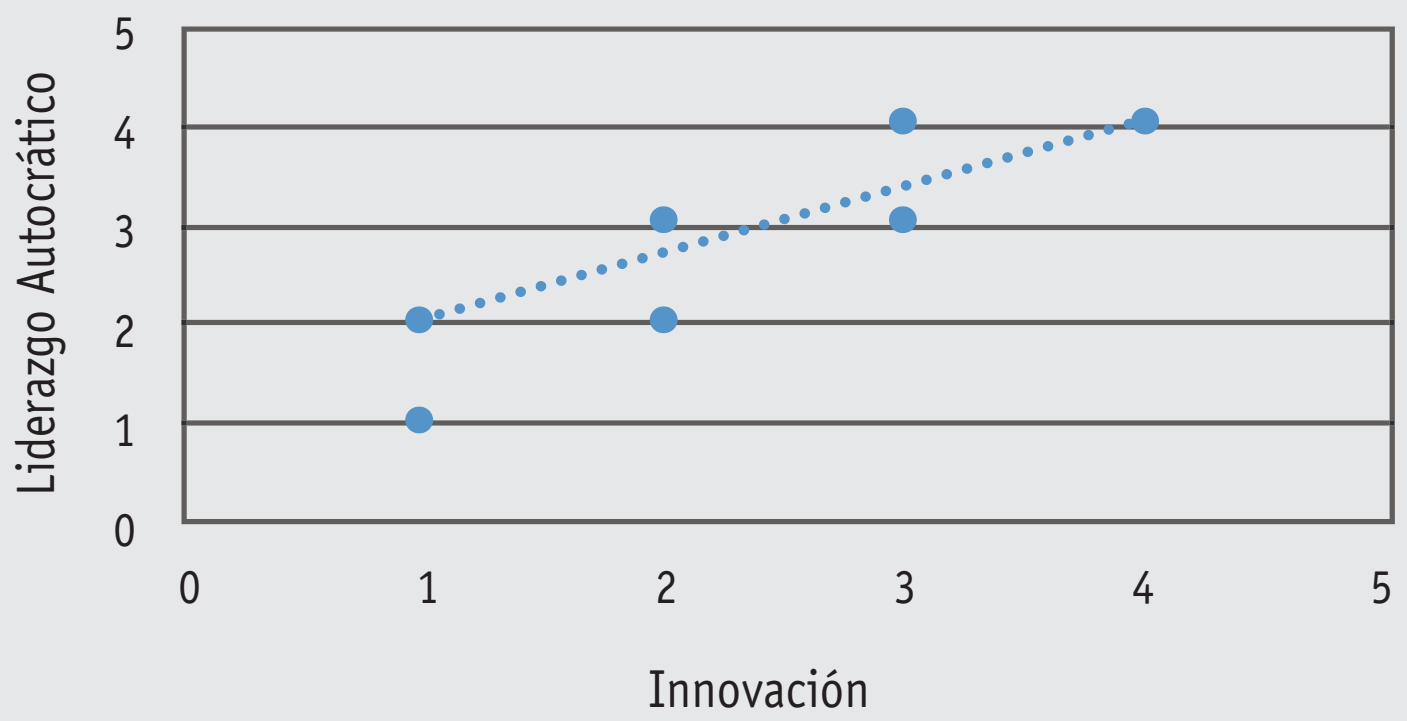

Fuente: elaboración propia.

Figura 3.

Liderazgo liberal

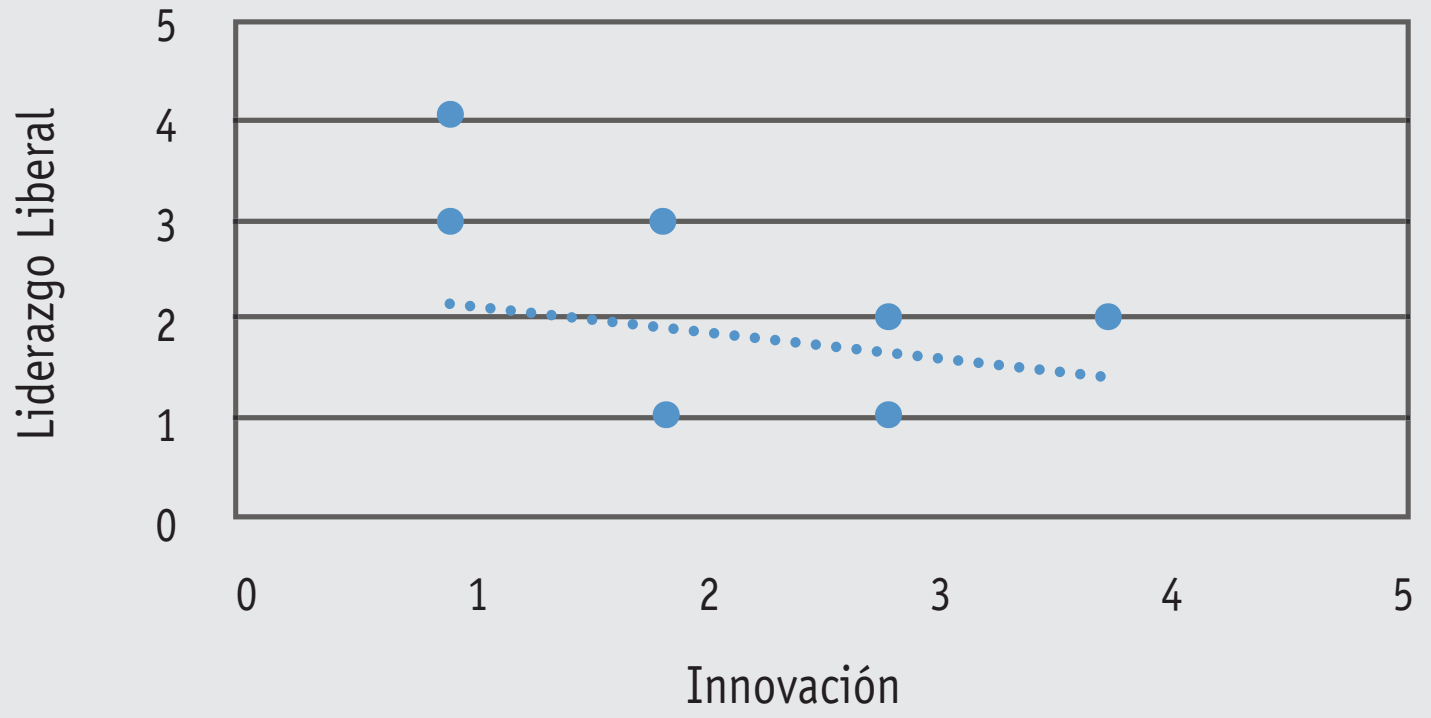

Fuente: elaboración propia. 


\section{DISCUSIÓN}

Es indiscutible que los estilos de liderazgo y las dinámicas de innovación son esenciales para las actividades y movimientos de los PYMES dentro de las nuevas fisionomías del mercado. Los empleados de las distintas empresas privadas de la comuna 11 de Medellín, a través de las encuestas, percibieron la necesidad de introducir unos elementos fundamentales para la organización empresarial. Por medio de la recolección de datos hallamos unos climas de innovación y liderazgo intermedios, pero que, sin duda, advierten unas transformaciones y transacciones imprescindibles para que los PYMES tomen disposición adecuada ante la economía global. $Y$ esto se sustenta, en primera instancia, con los trabajadores, pues como lo afirman Pons y Ramos (2012): "los miembros de una organización van configurando con el tiempo una visión homogénea de la misma" (p. 90). Esto define, entonces, que el líder debe adquirir nuevos fundamentos de innovación y liderazgo cuando el empleado se empodera de las dinámicas de la empresa.

Lo mencionado en el párrafo anterior evidencia que, a través de los datos expuestos en las tablas, es indispensable la exposición de la transformación a través del líder de la empresa, puesto que al momento del empleado sentir un deseo de cambio en su líder, aquel toma mayor confianza y su labor productiva es más eficaz, proporcionando un crecimiento individual y colectivo. Esto da cuenta, entonces, que el estilo mayor valorado en la Tabla 2 - estilo de liderazgo participativo - influye en las acciones de los empleados $y$, en consecuencia, denota una realización de objetivos y cumplimiento de los mismos.

\section{CONCLUSIÓN}

Es innegable la gran importancia que tiene el liderazgo para la consecución de las metas empresariales, la realización y adquisición de sentido de pertenencia de las personas que laboran en las compañías; el líder debe actuar como puente entre la alta dirección y los niveles operativos, para que la filosofía corporativa sea interiorizada y aplicada por cada uno de los integrantes de la organización. La innovación es consecuencia de un estilo de liderazgo participativo que permita la inclusión de todos los individuos en la creación o mejoramiento de productos y servicios que impacten la sociedad en un mundo globalizado

Ante el estudio realizado en los PYMES de la comuna 11 de Medellín se llega, además, a la conclusión que un líder debe adoptar y adaptar a sus funciones dentro de la organización empresarial unas habilidades imprescindibles con el propósito de que los miembros de la empresa se vinculen, de manera activa, a la constitución de metas económicas ofreciendo así un desarrollo satisfactorio y efectivo en pro del individuo y de la colectividad (empresa). Además, se profundizó que para un fortalecimiento del liderazgo en los PYMES, la comunicación-participación es trascendental para el vínculo líder-colaborador, ya que aquella ofrece, de manera indiscutible, una apuesta para la innovación; por medio del diálogo se consolidan grupos de trabajos que persiguen unos objetivos específicos.

Otro recurso relevante que se percibió en el estudio de los PYMES de la comuna 11 de Medellín fue la disposición del líder dentro de la empresa para equilibrarla y fortalecerla dentro del vertiginoso mundo del mercado global, por lo que es indispensable en el proceso de producción la participación del miembro de la empresa. Esto denota, entonces, una posición relevante del colaborador para el líder y la empresa; con el líder ofreciéndole voz a este, van construyendo ideas novedosas para un mejoramiento en la productividad. Este modelo autocrático de liderazgo proporciona seguridad, tranquilidad y paciencia para el miembro, reflejándose en sus procesos, en los objetivos alcanzados y en el crecimiento productivo.

En conclusión, los resultados obtenidos de este artículo de investigación con los PYMES de la comuna 11 de Medellín refuerzan y manifiestan el vínculo existente entre el estilo de liderazgo desarrollado por los PYMES, y la adopción de procesos que contribuyan a la adquisición de ideas innovadoras. Y, por encima de esta relación, debe tenerse presente, desde los distintos teóricos, que la generación de 
ideas innovadoras surge cuando los miembros de la organización perciben que el jefe les permite participar activamente y les capacita para desarrollar nuevas competencias; esto se debe a que las empresas se enfrentan a unas dinámicas más intensas; así como el que las reglas actuales de los mercados exijan que las organizaciones sean más competitivas e innovadoras, con el propósito de mantenerse en el mercado y sobresalir a través de la constitución de sus logros y proyectos. De manera tal que es indispensable la preparación y creación de nuevos funcionamientos pero sobretodo ser más visionarios, si es que quieren que el negocio se desarrolle.

\section{REFERENCIAS BIBLIOGRÁFICAS}

Berdecía, Z., González, J. y Carrasquillo, C. (2013). Estilos de liderazgo para el éxito organizacional estudios de casos múltiples en empresas puertorriqueñas. Revista de Estudios Avanzados de Liderazgo, 1(2), 21-32.

Escandon, D. y Hurtado, A. (2016). Influencia de los estilos de liderazgo en el desempeño de las empresas exportadoras colombianas. Estudios Gerenciales, 32(139), 137-145.
García Solarte, M. (2015). Formulación de un modelo de liderazgo desde las teorías organizacionales. Entramado, 11(1), 60-79.

González, J. y Carrasquillo, C. (2013). Estilos de liderazgo para el éxito organizacional: Estudio de casos múltiple para empresas puertorriqueñas. Revista de Estudios Avanzados de Liderazgo, Primavera, 1(2).

González, C. y Martínez, J.L. (2014). Gerencia estratégica e innovación empresarial: referentes conceptuales. Revista Dimensión Empresarial, 12(1), p. 107-116.

Hernández, R., Fernández, C. y Baptista, P. (2014). Metodología de la investigación. México: McGraw Hill.

Pons, V. F. y Ramos, L. J. (2012). Influencia de los Estilos de Liderazgo y las Prácticas de Gestión de RRHH sobre el Clima Organizacional de Innovación. Revista de Psicología del Trabajo y de las Organizaciones, 28(2), 81-98

Ramírez, M. G. (2013). Liderazgo organizacional. Un desafío permanente. Universidad \& Empresa, 15(25), 5-11.

Serrano, B. J. y Portalanza, A. (2014). Influencia del liderazgo sobre el clima organizacional. En: Suma de Negocios, 117-125.

Yeung, A., Ulrich, D., Nason, S. y Von, G. M. (2000). Las Capacidades de Aprendizaje en la Organización: Cómo aprender a Generar Ideas con Impacto. México: 0xford University Press. 